\title{
Real-World Outcomes of Advanced Soft Tissue Sarcoma Patients Treated with Pazopanib
}

Fawaz $A^{1 *}$, Shim $\mathbf{I}^{2}$, Tilley $\mathbf{D}^{2}$, Kelaney $\mathbf{M R}^{3}$, Abdelsalam OMA ${ }^{4}$ and Karachiwala $\mathrm{H}^{4}$

${ }^{1}$ Department of Medicine, University of Alberta, Canada ${ }^{2}$ Cancer Control, Alberta Heath Services, Canada

${ }^{3}$ Faculty of Medicine, Ain Shams University, Egypt ${ }^{4}$ Cross Cancer Institute, Edmonton, Alberta, Canada

*Corresponding author: Ali Fawaz, Department of Medicine, University of Alberta, Edmonton, Alberta, Canada

Received: March 22, 2021; Accepted: March 30, 2021; Published: April 06, 2021

\begin{abstract}
Background: Pazopanib is an oral multitarget tyrosine kinase inhibitor that is currently approved for the treatment of select subtypes of advanced Soft Tissue Sarcoma (STS) in patients who have progressed on prior anthracyclinebased chemotherapy regimens. In this study, we examine data from multiple centers to assess the efficacy of pazopanib in practice outside of a clinical trial setting.
\end{abstract}

Methods: A retrospective chart analysis was conducted for pre-treated, advanced soft tissue sarcoma patients who began treatment with pazopanib in Alberta, Canada and Cairo, Egypt (2012-2018).

Results: In total, 39 predominantly male (56.4\%) patients received pazopanib. The median age was $51,67 \%$ of whom had an ECOG of one or less. The predominant sarcoma subtype was leiomyosarcoma (30.8\%), and all patients had received at least one prior line of systemic therapy. Thirtytwo of the 39 patients (82\%) were initially given the full dose of $800 \mathrm{mg}$ with a median time on treatment of 116 days. Seven of the $39(18 \%)$ patients required a dose reduction while on treatment. A majority (94.9\%) of patients ultimately discontinued pazopanib treatment for reasons including death (21.6\%), disease progression (62.2\%), and toxicity (16.4\%). The median progression-free and overall survival for these patients was 4.1 months $(95 \% \mathrm{Cl}, 3.6-4.5)$ and 8.4 months $(95 \% \mathrm{Cl}, 4.3-12.5)$, respectively.

Conclusion: Pazopanib is an efficient and generally well-tolerated oral systemic therapy for the treatment of advanced, pre-treated, non-adipocytic soft tissue sarcoma. These results show the efficacy of pazoponib outside of a clinical trial setting.

Keywords: Pazopanib; Soft tissue sarcoma; STS; Real-world

\section{Introduction}

Soft Tissue Sarcomas (STS) are a subset of mesenchymal originating cancers, which are comprised of over 70 histologic subtypes and relatively rare with only 1025 cases diagnosed in Canada in 2016 [1]. Prognosis can be favourable for patients who present with a localized tumor that can be surgically resected. However, as many as $50 \%$ of patients will either present with or develop metastatic disease [2]. Those who develop metastasis have historically had limited therapeutic options, and despite recent advancements in therapy, have a poor overall prognosis with 5 years survival rates of $16 \%$ in Canada [3].

Currently, the gold-standard first-line therapy for metastatic disease is an anthracycline based regimen, most commonly with doxorubicin. For many years, patients whose disease progressed during first-line therapy were limited to either gemcitabine-based regimens or enrolment in a clinical trial. Recently, several landmark clinical trials have broadened the therapeutic landscape for the treatment of advanced STS with drugs such as trabectedin, eribulin and pazopanib having been approved in Canada and across the world for treatment of certain subtypes of advanced STS in patients with disease progression on prior lines of therapy [4-6]. These drugs, however, are not covered by most provincial funding bodies.

Pazopanib is an oral multitarget tyrosine kinase inhibitor. Its inhibitory action spans the Vascular Endothelial Growth Factor Receptor (VEGFR) and Platelet-Derived Growth Factor Receptor Alpha and Beta (PDGFRA/PDGFRB) [7]. Its efficacy in soft tissue sarcoma was demonstrated in the PALETTE trial, an international double-blinded randomized phase III control trial that compared pazopanib $(800 \mathrm{mg})$ to placebo in patients with non-adipocytic advanced STS who had progressed on previous lines of therapy [6]. This study demonstrated a statistically significant improvement in Progression-Free Survival (PFS) (4.6 months vs. 1.6 months) but not in Overall Survival (OS) (12.5 months vs. 10.7 months) in the pazopanib and placebo group, respectively. The most common toxicities experienced by patients were fatigue (49\%), diarrhea (16\%), nausea (28\%), weight loss (20\%), and hypertension (7\%).

Pazopanib is used worldwide for the treatment of advanced soft tissue sarcoma. However, in Canada, funding is not widely available resulting in challenges accessing the drug. We are interested in assessing the efficacy, safety, and uptake of pazopanib in a multiinstitutional review. The aim is to determine if pazopanib therapy has similar outcomes as historical controls and whether or not the
Sarcoma Res Int - Volume 6 Issue 1 - 2021

Submit your Manuscript | www.austinpublishinggroup.com

Fawaz et al. ( ) All rights are reserved
Citation: Fawaz A, Shim I, Tilley D, Kelaney MR, Abdelsalam OMA and Karachiwala H. Real-World Outcomes of Advanced Soft Tissue Sarcoma Patients Treated with Pazopanib. Sarcoma Res Int. 2021; 6(1): 1047. 
outcomes differ in a real-world setting.

\section{Methods}

The Alberta Cancer Registry identified 347 patients who were diagnosed with soft tissue sarcoma from 2012-2019 and received systemic therapy. Together with collaborative data collected from patients in Cairo, Egypt, 39 patients treated with pazopanib were identified. No patients receiving pazopanib were excluded.

From this data set, several patient characteristics were assessed including median age, sex, location of treatment, initial Eastern Cooperative Oncology Group (ECOG) performance status,

Table 1: Baseline patient characteristics.

\begin{tabular}{|c|c|c|}
\hline & N (39) & $\%$ \\
\hline \multicolumn{3}{|l|}{ Age } \\
\hline Median (range) & $51(20-68)$ & \\
\hline \multicolumn{3}{|l|}{ Sex } \\
\hline Male & 22 & 56.4 \\
\hline Female & 17 & 43.6 \\
\hline \multicolumn{3}{|l|}{ Location } \\
\hline Calgary & 13 & 33.3 \\
\hline Edmonton & 15 & 38.5 \\
\hline Cairo & 11 & 28.2 \\
\hline \multicolumn{3}{|l|}{ ECOG at Start } \\
\hline Zero & 4 & 11.4 \\
\hline One & 22 & 62.9 \\
\hline Two & 9 & 25.7 \\
\hline Unknown & 4 & NA \\
\hline \multicolumn{3}{|l|}{ Pathological Diagnosis } \\
\hline Leiomyosarcoma & 12 & 30.8 \\
\hline MPNST (Nerve Shealth) & 3 & 7.7 \\
\hline Angiosarcoma & 2 & 5.1 \\
\hline SS & 2 & 5.1 \\
\hline UPS & 3 & 7.7 \\
\hline Synovial & 4 & 10.3 \\
\hline Other & 13 & 33.3 \\
\hline \multicolumn{3}{|l|}{ Prior systemic lines } \\
\hline One & 19 & 48.7 \\
\hline Two & 18 & 46.2 \\
\hline Three & 2 & 5.1 \\
\hline \multicolumn{3}{|l|}{ Prior Doxorubicin } \\
\hline Yes & 37 & 94.9 \\
\hline No & 2 & 5.1 \\
\hline \multicolumn{3}{|c|}{ Prior Radiotherapy (before Pazopanib) } \\
\hline Yes & 28 & 71.8 \\
\hline No & 11 & 28.2 \\
\hline \multicolumn{3}{|l|}{ Prior Surgery } \\
\hline Yes & 23 & 59 \\
\hline No & 16 & 41 \\
\hline
\end{tabular}

pathologic diagnosis, prior systemic lines, prior treatment with surgery or radiotherapy, method of drug funding, starting dose, dose modifications, the median time on treatment, drug toxicities and subsequent treatments.

Kaplan-Meier estimates were made to calculate median progression-free and overall survival.

\section{Results}

A total of 39 patients ( 22 male, 17 female) were reviewed for this study. Their baseline characteristics are displayed in Table 1. Eleven of the patients were from the Cairo cohort, while the remaining 28 were from Alberta. The median age was 51 (range, 20-68). Twentysix (67\%) patients had an ECOG performance status of 1 or less. The predominant pathological diagnosis was leiomyosarcoma (30.8\%). All patients had been treated previously with systemic chemotherapy, with $48.7 \%$ receiving one previous line of chemotherapy and $46.2 \%$ receiving two. Almost all of the patients had been treated previously with doxorubicin (94.9\%). A majority had been treated with radiotherapy $(71.8 \%)$ and/or surgery $(59.0 \%)$ prior to pazopanib initiation.

Patients who initiated pazopanib between 2012 and 2019 are shown in Table 2. Most patients began therapy at the standard dose of $800 \mathrm{mg}$ once daily $(82.1 \%)$. Seven patients $(17.9 \%)$ required a dose reduction and two (5.1\%) had their dose increased while on therapy. At the time of review, the vast majority of patients had discontinued the drug (94.9\%) (Table 3). The major reason for cessation of treatment was disease progression (62.2\%). Eight patients (21.6\%) had passed away, and six patients (16.2\%) had ceased due to toxicity. Of those six patients, two had cardiac-related toxicity, one had Table 2: Dosing regimen and modifications.

\begin{tabular}{|c|c|c|}
\hline & N (39) & $\%$ \\
\hline \multicolumn{3}{|l|}{ Year Start } \\
\hline 2012 & 2 & 5.1 \\
\hline 2013 & 4 & 10.3 \\
\hline 2014 & 4 & 10.3 \\
\hline 2015 & 4 & 10.3 \\
\hline 2016 & 4 & 10.3 \\
\hline 2017 & 12 & 30.8 \\
\hline 2018 & 7 & 17.9 \\
\hline 2019 & 2 & 5.1 \\
\hline \multicolumn{3}{|l|}{ Starting Dose } \\
\hline 800 & 32 & 82.1 \\
\hline 600 & 3 & 7.7 \\
\hline 400 & 3 & 7.7 \\
\hline 200 & 1 & 2.6 \\
\hline \multicolumn{3}{|l|}{ Dose Modification } \\
\hline Any & 9 & 23.1 \\
\hline Reduction & 7 & 17.9 \\
\hline$\%$ Initial Dose Median (range) & \multicolumn{2}{|c|}{$66.6 \%(25-75 \%)$} \\
\hline Increase & 2 & 5.1 \\
\hline \% Initial Dose Median (range) & \multicolumn{2}{|c|}{$200.0 \%(200-200 \%)$} \\
\hline
\end{tabular}


Table 3: Reasons for stopping pazopanib and subsequent therapies.

\begin{tabular}{|l|c|c|}
\hline & N (39) & $\%$ \\
\hline Treatment Status & & \\
\hline Still on drug & 2 & 5.1 \\
\hline Drug halted & 37 & 94.9 \\
\hline Reason drug halted & & \\
\hline Death & 8 & 21.6 \\
\hline Disease progression* & 23 & 62.2 \\
\hline Toxicity & 6 & 16.2 \\
\hline Cardiac & 2 & 66.6 \\
\hline Hypertension & 1 & 33.3 \\
\hline Unspecified toxicity & 3 & NA \\
\hline Subsequent Therapy (n= 29 eligible) & & \\
\hline None & 19 & 65.5 \\
\hline Ifosfamide & 4 & 13.8 \\
\hline Gem/Doce & 3 & 10.3 \\
\hline Yondelis & 1 & 3.4 \\
\hline Trial (CTM2 phase I) & 1 & 3.4 \\
\hline Dacarbazine & 3.4 \\
\hline Not 1 pati & 1 & \\
\hline
\end{tabular}

${ }^{*}$ Note: 1 patient lost access to drug for 5 months due to drug shortage, but did resume drug.

Table 4: Time on treatment.

\begin{tabular}{|c|c|c|}
\hline & $N(39)$ & $\%$ \\
\hline \multicolumn{3}{|c|}{ Time on Treatment (days) } \\
\hline Median (range) & \multicolumn{2}{|c|}{$116(12-543)$} \\
\hline$<30$ days & 4 & 10.3 \\
\hline 30-90 days & 9 & 23.1 \\
\hline 91-180 days & 15 & 38.5 \\
\hline 181-365 days & 3 & 7.7 \\
\hline >365 days & 8 & 20.5 \\
\hline
\end{tabular}

hypertension, and three were unspecified. The total time each patient spent on pazopanib can be seen graphically in Figure 1. The median time on therapy was 116 days (range, 12-543) (Table 4).

Table 5 shows how pazopanib was funded for the patients in the study. Funding sources were fairly evenly distributed between Cancer Institute funding (23.7\%), compassionate care (23.7\%), private insurance $(26.3 \%)$ and national insurance $(26.3 \%)$.

The Kaplan-Meier survival curves can be seen in Figure 2 and 3. The median overall survival for the whole cohort was 8.4 months (95\% CI, 4.3 - 12.5). Specifically, the Alberta cohort had a median OS of 8.4 months (95\% CI, 6.2 - 10.7), while the Cairo cohort had a median OS of 13 months (95\% CI, 5.8 - 20) (Table 6). The median progression-free survival for the whole cohort was 4.1 months $(95 \%$ CI, 3.6 - 4.5).

\section{Discussion}

This study aimed to examine real-world patient characteristics and outcomes for the use of pazopanib in advanced non-adipocytic soft tissue sarcoma in Alberta, Canada and Cairo, Egypt. To our

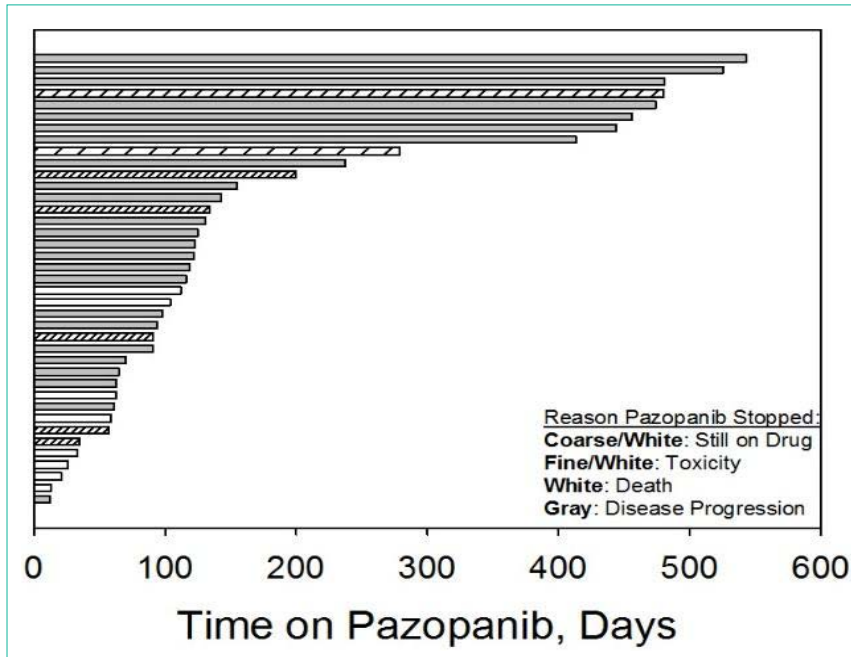

Figure 1: Time on pazopanib for each patient along with the reason for stopping the medication. Graphic generated via SigmaPlot v13.0.

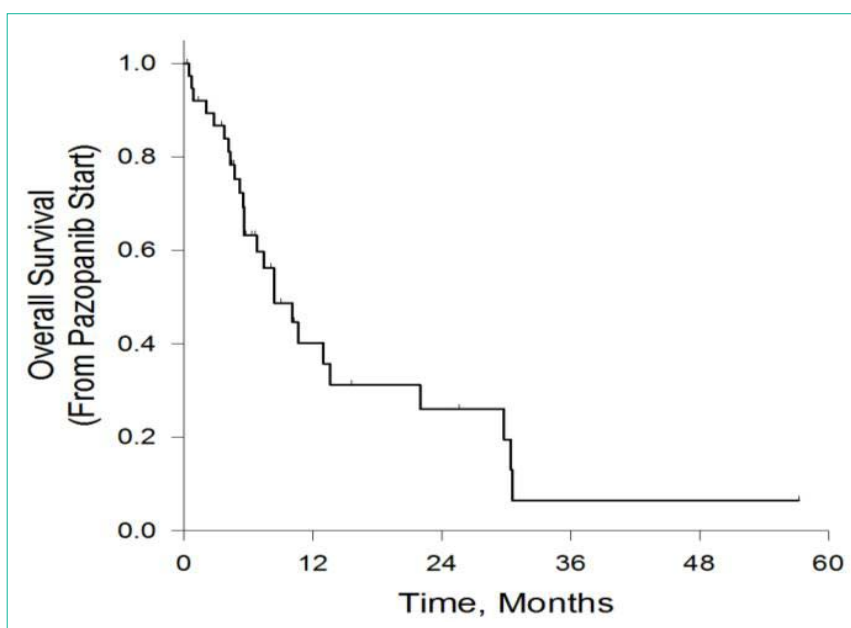

Figure 2: Kaplan-Meier curve for overall survival. Median overall survival was $8.4 \mathrm{~m}(95 \% \mathrm{Cl}, 4.3-12.5)$. Graphic generated via SigmaPlot v13.0.

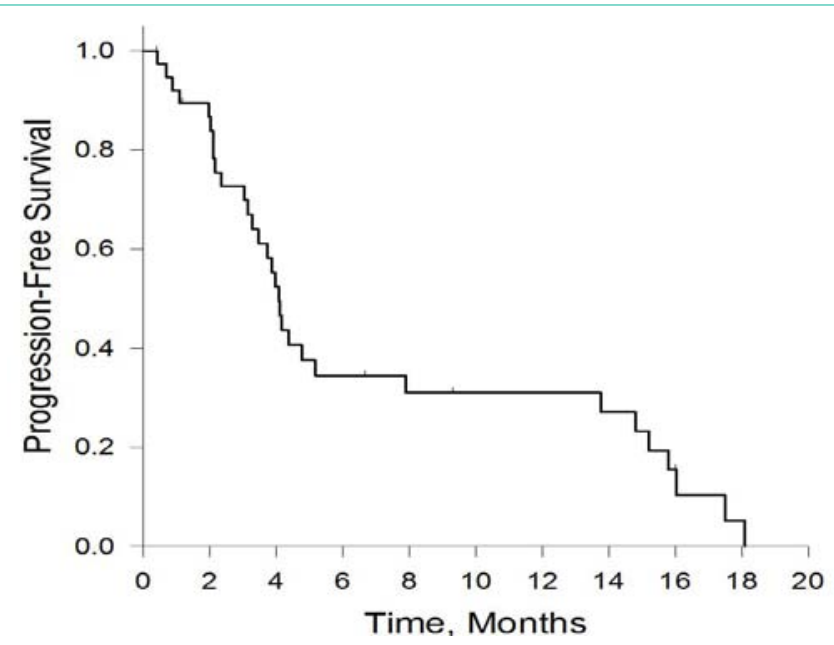

Figure 3: Kaplan-Meier curve for progression-free survival. Median progression-free survival $4.1 \mathrm{~m}(95 \% \mathrm{Cl}, 3.6-4.5)$. Graphic generated via SigmaPlot v13.0. 
Table 5: Funding source.

Table 5: Funding source.
\begin{tabular}{|l|c|c|}
\hline & N (39) & $\%$ \\
\hline Drug Funding & & \\
\hline Cancer Institute Funding & 9 & 23.7 \\
\hline Compassionate Care & 9 & 23.7 \\
\hline Private Insurance & 10 & 26.3 \\
\hline National Insurance & 10 & 26.3 \\
\hline Unknown & 1 & NA \\
\hline
\end{tabular}

Table 6: Median overall survival for the cohorts individually and as a whole.

\begin{tabular}{|l|c|}
\hline Cohort & Median OS (95\% Cl) \\
\hline Whole cohort & $8.4(4.3-12.5)$ \\
\hline Alberta Only & $8.4(6.2-10.7)$ \\
\hline Cairo Only & $13(5.8-20)$ \\
\hline
\end{tabular}

knowledge, this is the first study to examine pazopanib use in these populations.

The major outcomes examined were median progression-free and overall survival. The progression-free survival of 4.1 months $(95 \% \mathrm{CI}$, 3.6 - 4.5) is similar to the 4.6 months reported in the pazopanib group of the PALETTE clinical trial [6]. However, we report a lower median OS of 8.4 months (95\% CI, 4.3-12.5) compared to 12.5 months observed in the clinical trial. In comparing the study populations, a number of key differences arise that might explain the lower OS observed in our study. Firstly, the majority of our patients had an ECOG performance status of 1 or greater (89\%), with 25\% having a performance status of 2 . Comparatively, $46 \%$ of the pazopanib treatment group in the PALETTE study had a performance status of 0 and none greater than 1 . Unsurprisingly, the PALETTE study found that a performance status of 0 compared to 1 was associated with a hazard ratio of 0.72 (95\% CI, 0.53 - 0.97) for Progression-Free Survival (PFS), which was statistically significant. Another potentially important difference between our populations was the extent to which participants received chemotherapy after progression on pazopanib. Greater than $50 \%$ of participants in the pazopanib treatment group in the PALETTE trial received post-protocol systemic therapies. By contrast, only $34.4 \%$ of eligible patients in our study group received further systemic therapies after progressing on pazopanib. In our populations, funding limitations for subsequent lines (i.e. third line or greater) of systemic therapy was likely a major contributor to discrepancies in the extent to which post-pazopanib therapy was initiated. A final, albeit likely smaller contributor to the lower median OS observed in our population could have been the starting pazopanib dose. A small subset of the patients studied (18\%) were initiated on pazopanib at a dose below the standard of $800 \mathrm{mg}$ daily.

Since the publication of the PALETTE clinical trial, there have been a number of real-world retrospective analyses for pazopanib use in centers around the world. A wide range of median OS is reported in these studies from as low as 8.2 months to as high as 18 months [813]. The wide range in reported median OS highlights one of the main challenges in studying a rare and heterogeneous subset of cancers, such as soft tissue sarcoma. For example, while the predominant pathologic diagnosis for most of the studies was leiomyosarcoma, one study looking at Indian patients identified synovial sarcoma as the predominant subset [11]. That same study also demonstrated the longest median OS of 18 months. Furthermore, wide variation exists in the baseline performance status of the patients being studied, frequency of dose alterations, and the extent to which patients were treated prior to pazopanib initiation, as well as after progression on pazopanib. These differences are particularly exacerbated by the rarity of these cancers and the resulting difficulty in accruing large sample sizes for analysis. Of the studies mentioned, the number of patients analyzed were generally low, ranging from 33 to 211 . Ultimately, while the median OS in our study is on the lower end of those determined in previous studies, for the above reasons, it can be challenging to make direct comparisons.

In our study, we determined the median time on pazopanib to be 116 days (range, 12-543). This is in line with the median treatment duration of 16.4 weeks (114.8 days) in the pazopanib treatment group of the PALETTE trial [6]. Furthermore, only a minority of our patients discontinued therapy due to toxicity. This, together with the fact that a dose reduction was required in only seven patients (18\%), suggests that pazopanib was generally well tolerated.

One of the key challenges in studying rare tumours such as soft tissue sarcomas is obtaining a sufficient sample size of patients from which to derive meaningful data. We attempted to address this issue in our study by supplementing the local Alberta cohorts, with a cohort of patients from Cairo, Egypt. While this did allow a larger sample size for analysis, we do appreciate that this approach is limited by the fact that we are analyzing two distinct patient populations with varying clinical characteristics as a single group. Regardless, we feel this is an acceptable concession, as there remains a significant need to understand the efficacy and tolerability of these novel therapies in this rare tumour group.

Funding considerations remain a significant barrier to pazopanib use in our centers. At the time of writing, pazopanib is approved by Health Canada but is not provincially funded in the majority of Canadian provinces, including Alberta. As a result, all of the patients from our Alberta cohort had pazopanib funded through either compassionate care, Cancer Institute funding, or private insurance. Despite the limitations discussed, we feel the present study nevertheless makes a case for pazopanib as an efficient, generally welltolerated systemic alternative for the treatment of non-adipocytic, pre-treated advanced STS that should receive consideration for universal access. This notion is further supported by several of the previously mentioned retrospective analyses performed in diverse patient populations across the world. Furthermore, although none have yet been established, there is active work being done to identify clinico-pathologic, radiologic, pharmacodynamic and biological markers that can be used to better identify patients who would most benefit from targeted agents such as pazopanib [14]. This patientcentered approach may make pazopanib and other targeted systemic agents more effective in the future.

\section{Conclusion}

This retrospective analysis looked at real-world pazopanib use in multiple centers. While we report a medium overall survival that is on the lower end of historical controls, the medium progression-free survival is similar to what has previously been reported. Furthermore, we found pazopanib to be generally well tolerated by the populations 
studied. As such, we believe pazopanib is a viable treatment option for patients with pre-treated, non-adipocytic STS, and should be considered for universal funding.

\section{Declarations}

\section{Availability of data and material}

All data generated or analyzed during this study are included in this published article.

\section{Contributions}

Hatim Karachiwala and Omar Abdelsalam designed the study. Mohamed Kelaney collaborated and provided data from his institution. Isaac Shim and Derek Tilley compiled the data, generated the figures and tables, and performed statistical analysis. Ali Fawaz wrote the manuscript. All listed authors were involved in editing and critical review of the manuscript.

\section{Ethics approval}

This research was approved by the Ethics Board of AlbertaCancer Committee effective December 16, 2019.

Consent to participate Due to the nature of this study consent could not practically be obtained. As such this requirement was waived as per the above ethics board approval.

\section{References}

1. Soft tissue sarcoma statistics - Canadian Cancer Society. 2020

2. George S. Developments in Systemic Therapy for Soft Tissue and Bone Sarcomas. Journal of the National Comprehensive Cancer Network. 2019; 17: $625-628$

3. Survival statistics for soft tissue sarcoma - Canadian Cancer Society. 2020.

4. Schöffski P, Chawla S, Maki RG, Italiano A, Gelderblom H, Choy E, et al Eribulin versus dacarbazine in previously treated patients with advanced liposarcoma or leiomyosarcoma: A randomised, open-label, multicentre, phase 3 trial. In: The Lancet. Lancet Publishing Group. 2016; 387: 1629 1637.
5. Demetri GD, von Mehren M, Jones RL, Hensley ML, Scott M, Schuetze, et al. Efficacy and safety of trabectedin or dacarbazine for metastatic liposarcoma or leiomyosarcoma after failure of conventional chemotherapy: Results of a phase III randomized multicenter clinical trial. Journal of Clinical Oncology. 2016; 34: 786-793

6. van der Graaf WTA, Blay JY, Chawla SP, Kim DW, Bui-Nguyen B, Casali PG, et al. Pazopanib for Metastatic Soft-Tissue Sarcoma (PALETTE): A randomised, double-blind, placebo-controlled phase 3 trial. The Lancet. 2012; 379: 1879-1886.

7. Schutz FAB, Choueiri TK, Sternberg CN. Pazopanib: Clinical development of a potent anti-angiogenic drug. Critical Reviews in Oncology/Hematology. 2011; $77:$ 163-171.

8. Gelderblom H, Judson IR, Benson C, Grignani G, Katz D, Klaus W, et al. Treatment patterns and clinical outcomes with pazopanib in patients with advanced soft tissue sarcomas in a compassionate use setting: results of the SPIRE study. Acta Oncologica. 20107; 56: 1769-1775.

9. Yoo KH, Kim HS, Lee SJ, Park SH, Kim SJ, Kim SH, et al. Efficacy of pazopanib monotherapy in patients who had been heavily pretreated for metastatic soft tissue sarcoma: a retrospective case series. BMC Cancer. 2015; 15: 154.

10. Seto T, Song M-N, Trieu M, Yu J, Sidhu M, Liu CM, et al. Real-World Experiences with Pazopanib in Patients with Advanced Soft Tissue and Bone Sarcoma in Northern California. Medical Sciences. 2019; 7: 48.

11. Sharma A, Vanidassane I, Aggarwal A, Mridha AR, Pandey R, Dhamija E, et al. Pazopanib efficacy and toxicity in a metastatic sarcoma cohort: Are Indian patients different? Indian journal of cancer. 2019; 56: 207-210.

12. Nakamura T, Matsumine A, Kawai A, Araki N, Goto T, Yonemoto T, et al. The clinical outcome of pazopanib treatment in Japanese patients with relapsed soft tissue sarcoma: A Japanese Musculoskeletal Oncology Group (JMOG) study. Cancer. 2016; 122: 1408-1416.

13. Koca S, Beşiroglu M, Ozcelik M, Karaca M, Bilici M, Haciogiu B, et al. Pazopanib for metastatic soft-tissue sarcoma: A multicenter retrospective study. Journal of Oncology Pharmacy Practice. 2020; 107815522092407.

14. Lee ATJ, Jones RL, Huang PH. Pazopanib in advanced soft tissue sarcomas. Signal Transduction and Targeted Therapy. 2019; 4: 16. 